\title{
Accesibilidad web en universidades del consejo de rectores de Chile: análisis preliminar
}

\author{
Yorka Tatiana Ortiz Ruiz - Universidad de Los Lagos
}

$0000-0002-2870-2253$

Recepción: 24.07.2020 | Aceptado: 30.10.2020

Correspondencia a través de ORCID: Yorka Tatiana Ortiz Ruiz

(iD 0000-0002-2870-2253

Citar: Ortiz Ruiz, YT (2020). Accesibilidad web en universidades del consejo de rectores de Chile: análisis preliminar. REIDOCREA, 9, 220-231.

\begin{abstract}
Resumen: El presente estudio tiene como objetivo evaluar el nivel de accesibilidad de los sitios web iniciales de las universidades que formar parte del denominado Consejo de Rectores de Chile, veintisiete universidades estatales y públicas no estatales considerando no existe información actualizada al respecto y la inclusión es un tema que nos involucra a todos. Existen leyes y una guía de accesibilidad web actualizada el año 2017, del Servicio Nacional de la Discapacidad que establece el nivel AA como exigencia de accesibilidad, elaborada en base a las pautas de accesibilidad para contenidos web (WCAG) 2.0 del World Wide Web Consortium (W3C). A partir de estas referencias se evaluaron de forma preliminar, usando herramientas automáticas especializadas como son TAW y eXaminator, las páginas de inicio o home de cada universidad. Todos resultaron ser sitios no accesibles con problemas, advertencias y no verificables, siendo las debilidades más recurrentes la ausencia de textos alternativos en imágenes, videos u otros elementos que lo exigen, enlaces idénticos con destinos diferentes, entre otros errores que son exigencias básicas.
\end{abstract}

Palabra clave: Educación

\section{Web accessibility in universities of the council of rectors of Chile: preliminary analysis}

\begin{abstract}
The objective of this study is to evaluate the level of accessibility of the initial websites of the universities that form part of the so-called Council of Rectors of Chile, twenty-seven state and non-state public universities considering that there is no updated information about this, and inclusion is an issue that involves all of us. There are laws and a web accessibility guide updated in 2017 by the National Disability Service that establishes the AA level as a requirement for accessibility, based on the accessibility guidelines for web content (WCAG) 2.0 of the World Wide Web Consortium (W3C). From these references were evaluated in a preliminary way the home pages of each university using specialized automatic tools such as TAW and eXaminator. All proved to be inaccessible sites with problems, warnings and unverifiable, the most recurrent weaknesses being the absence of alternative texts in images, videos or other elements that require it, identical links with different destinations, among other errors that are basic requirements.
\end{abstract}

Keyword: Education

\section{Introducción}

Existe acuerdo en señalar que Internet es la principal fuente de información en la actualidad en todo ámbito del quehacer humano, uno de los más influenciados es el educativo. Es así que a nivel universitario se difunde la información vía internet, en específico sus sitios web, sobre carreras, requisitos, becas, contacto, organización, entre tantos otros temas propios de la formación profesional y formación continua. En Chile existen 61 universidades $^{1}, 27$ de la cuales forman parte del denominado Consejo de Rectores de las Universidades Chilenas (Cruch), se trata de universidades estatales y públicas no estatales del país, cada una de ellas de amplia trayectoria y excelencia en Educación Superior, con presencia en todas las regiones de Chile. ${ }^{2}$

\footnotetext{
${ }^{1}$ Disponible en: http://www.webometrics.info/es/Latin America es/Chile

${ }^{2}$ Extraído de: http://www.consejoderectores.cl/universidades
} 
Considerando la relevancia de Internet en todo campo de acción es de gran responsabilidad disponer de espacios en línea que garanticen la accesibilidad de todo usuario, en especial aquellos sitios desarrollados por instituciones educativas, considerando que el acceso a la información es un derecho de todos y todas. De esta forma se realizará un análisis de las políticas actuales relacionadas con accesibilidad usando las tecnologías lo cual se verificará con la evaluación de los sitios iniciales o home de las universidades pertenecientes al Consejo de Rectores o denominadas Universidades Tradicionales, para esto se trabaja usando como referencia la guía de accesibilidad web realizada por el Servicio Nacional de la Discapacidad (SENADIS). Esta guía señala que en Chile, existen $2.119 .316^{3}$ personas con una o más discapacidades, por lo que el Servicio Nacional de la Discapacidad (SENADIS, 2018), recomienda trabajar en el tema para disminuir las barreras de accesibilidad y permitir que todos y todas podamos acceder de igual manera a la información.

Este análisis cobra especial relevancia si consideramos el impacto de Internet en la sociedad y en específico en Chile, que según el Ranking "The Inclusive Internet Index 2018", ranking elaborado por The Economist Intelligence Unit y patrocinado por Facebook, Chile es el primer país latinoamericano y el octavo a nivel global en acceso y uso de internet (I. Barberis, 2018).

Según lo señalado por San_Anlas y Stable - Rodríguez (2016) la accesibilidad web es una métrica de la Ingeniería Web que trata de elementos relacionados con la codificación y la presentación de información en el diseño y funcionalidad de un sitio web, que va a permitir que las personas con algún tipo de discapacidad puedan percibir, entender, navegar e interactuar de forma efectiva en la web, así como crear y aportar contenido. Su incumplimiento ocasiona discriminación debido a que no pueden utilizar la información disponible en la web de manera normal.

Otras entidades definen accesibilidad como la cualidad de ser accesible, la Real Academia Española la define como "que tiene acceso", "de fácil acceso o trato" o "de fácil comprensión, inteligible”. (RAE, 2018)

Según el observatorio de la accesibilidad4 (COCEMFE, 2018), esta se puede definir como la cualidad de fácil acceso para que cualquier persona - incluso aquellas que tengan limitaciones en la movilidad, en la comunicación o el entendimiento - pueda llegar a un lugar, objeto o servicio.

Para la Organización Nacional de Ciegos Españoles (ONCE, 2018), la accesibilidad es la condición que deben cumplir los entornos, procesos, bienes, productos y servicios, así como los objetos 0 instrumentos, herramientas y dispositivos para ser comprensibles, utilizables y practicables por todas las personas en condiciones de seguridad y comodidad; y de la forma más autónoma y natural posible.

El Seminario Iberoamericano sobre Diversidad y Accesibilidad en la Red (Sidar, 2018) establece principios del denominado Diseño Universal o Diseño para Todos, en que menciona se centran en el diseño utilizable universalmente o por todos, pero hay que tener en cuenta que en el diseño intervienen otros aspectos, como el coste, la cultura en la que será usado, el ambiente, etc.; que tampoco pueden olvidarse. Estos principios generales del diseño, son aplicables y de hecho se aplican en la arquitectura, la

\footnotetext{
3 "Censo 2012 en Discapacidad revela que las personas con discapacidad son el principal 'grupo vulnerable' en Chile" [online]. Disponible en www.senadis.gob.cl

${ }^{4}$ Disponible en: https://www.observatoriodelaaccesibilidad.es/
} 
ingeniería y, por supuesto, las páginas y aplicaciones Web, entre otros campos de aplicación.

El World Wide Web Consortium (W3C, 2018a) establece que hablar de Accesibilidad Web es hablar del acceso de todos a la Web, independientemente del tipo de hardware, software, infraestructura de red, idioma, cultura, localización geográfica y capacidades de los usuarios.

El Portal de las Personas con Discapacidad (Discapnet, 2018) entiende por "accesibilidad de portales Web" el conjunto de tecnologías, normas de aplicación y diseño que facilitan la utilización de los sitios Web siguiendo las pautas del "diseño para todos". El fundamento para desarrollar una web accesible radica en comprender que las personas acceden a Internet de modos diferentes:

- Usuarios con ceguera total que usan un programa lector de pantalla para acceder al contenido del navegador, escuchándolo por los altavoces o leyéndolo con sus dedos mediante una línea Braille.

- Usuarios con deficiencia visual, visión parcial o ceguera al color, que emplean sistemas de ampliación de la pantalla, recurren al aumento del tamaño de las letras o requieren un contraste efectivo de color entre el texto y el fondo.

- Usuarios con limitación motriz en sus manos, que no pueden utilizar el ratón y acceden al contenido web exclusivamente mediante el teclado de su ordenador o lo hacen mediante sistemas de reconocimiento de voz, navegando mediante instrucciones verbales que dan a su ordenador.

- Usuarios con sordera total que requieren de alternativas textuales a elementos multimedia sonoros.

- Pero también hay usuarios de conexiones lentas a Internet o que acceden mediante equipos portátiles del tipo "palm top" o teléfonos móviles con reducidas pantallas gráficas, que se benefician del diseño accesible.

Estas definiciones de diversas entidades dejan claro que el concepto de accesibilidad, además de ser un término recurrente en sus objetivos, está respaldado por el denominado Diseño para Todos o Diseño Universal y busca atender la diversidad funcional como algo inherente al ser humano y sus capacidades diversas en el transcurso de la vida, es decir es un aspecto que no solo busca acercar la información a personas con algún tipo de discapacidad, sino también a personas que ven deterioradas sus capacidades a lo largo de la vida o pasan por alguna situación desfavorable de salud, siendo una constante el que debe considerar el acceso no solo a espacios físicos, sino también a información disponible en todo formato, muy relevante es el acceso a medios electrónicos en línea, los cuales se actualizan constantemente y son el medio de comunicación y difusión por excelencia.

Un concepto muy relevante que se mencionó anteriormente es el de diversidad funcional, en el que se ahonda por ser la circunstancia de ser distinto, de no ser uniforme - único. Cada persona es diferente, diferentes sus características, realidades, necesidades y limitaciones. El hecho de que cada ciudadano tenga esta condición como ser único y diferente, confiere el carácter de diversa a la sociedad.....La forma de moverse, pensar, sentir o percibir, es diferente entre individuos, algo que, en sí mismo, no es un problema. Éste surge cuando estas diferencias se alejan del modelo "normal" o "común" y la persona no puede utilizar los mismos sistemas o esquemas que el resto de sus conciudadanos. En ese caso es cuando aparece la discapacidad. Esto ocurre desde el nacimiento y a edades tempranas; pero también a lo largo de la vida, por accidentes, emigración, enfermedades; y de forma más notoria, al avanzar la edad y envejecer (Pastor, 2012) 
Dado los antecedentes presentados, es importante que los sitios web cumplan con estándares de accesibilidad para lograr que un mayor número de usuarios los visiten sin contratiempos, estándares que en muchos países han sido desarrollados con el respaldo estatal, es así que el objetivo de esta investigación consistió en revisar la legislación junto con evaluar la accesibilidad web en forma preliminar de sitios de ingreso de las 27 universidades del consejo de rectores.

\section{Objetivo}

Evaluar de forma preliminar, usando herramientas automáticas especializadas como son TAW y eXaminator, las páginas de inicio o home de cada universidad del denominado Consejo de Rectores de Chile, veintisiete universidades estatales y públicas no estatales.

\section{Métodos}

\section{Enfoque de Investigación:}

Cuantitativo, considerando que es un proceso secuencial y probatorio. Se trata de determinar la fuerza de asociación o correlación entre variables, la generalización y objetivación de los resultados a través de una muestra para hacer inferencia a una población de la cual toda muestra procede. (Fernández \& Pértegas, 2002)

El análisis de accesibilidad de las páginas iniciales de las 27 Universidades del Consejo de Rectores de Chile se realizó desde el 26 de septiembre al 03 de Octubre de 2019, tomando como base las recomendaciones técnicas que el Servicio Nacional de la Discapacidad establece en su guía de accesibilidad Web, las cuales se basan en los lineamientos establecidos en las Pautas de Accesibilidad para el Contenido Web (WCAG) 2.0, publicadas el 11 de diciembre de 2008 por la Iniciativa para la Accesibilidad Web (WAI) del World Wide Web Consortium (W3C). Éstas fueron aprobadas como estándar internacional ISO/IEC 40500:2012, el 15 de octubre de 2012.

Esta Guía de Accesibilidad web en Chile señala que la accesibilidad se ha introducido fuertemente en el uso de Internet y en relación a ello, el World Wide Web Consortium (W3C, 2018a) publicó las Pautas de Accesibilidad para Contenidos Web (WCAG) 2.0, material validado y utilizado como marco de referencia para la adopción de medidas, por ejemplo, en Estados Unidos, la Comunidad Europea, Japón, México, Argentina, e instituciones de y para personas en situación de discapacidad visual -como la Organización Nacional de Ciegos de España (ONCE, 2018) y desde 2015 en Chile con el Decreto №1 del ministerio Secretaría General de la Presidencia, entre otras innumerables organizaciones de la sociedad civil que han velado por la universalidad del uso de la informática.

\section{Herramientas de análisis}

Para la evaluación de la accesibilidad web se han utilizado herramientas disponibles que permiten automatizar este proceso, según las pautas WCAG 2.0.

Las herramientas automáticas de evaluación de la accesibilidad web son programas de software o servicios en línea, que realizan una inspección automática para ayudar a determinar si un sitio web satisface las guías o normas de accesibilidad web. Existen numerosas herramientas automáticas para la evaluación de la accesibilidad web (Luján 
Mora, 2018), pero considerando las definiciones establecidas desde el Servicio Nacional de la Discapacidad y sus recomendaciones, se ha optado por la herramienta automatizada TAW (CTIC, 2018) y eXaminator como una forma de contrastar los resultados.

La herramienta TAW de evaluación de la accesibilidad web desarrollada por la Fundación CTIC (Centro Tecnológico de la Información y la Comunicación), sede del W3C en España, está en línea desde abril de 2001 y fue el primer verificador de la accesibilidad web en español. TAW evalúa de forma automática el nivel de conformidad de las pautas de la WCAG 1.0 y WCAG 2.0, pero como se mencionó anteriormente el estudio se remite a las pautas WCAG 2.0. La primera genera un reporte de salida en el que aparecen los resultados del análisis, indicando el número de problemas detectados, en relación al nivel de prioridad del criterio de verificación correspondiente a la guía con la que se evalúa. Estos informes de evaluación no son definitivos, pero constituyen una primera etapa en el proceso de revisión. La segunda herramienta (eXaminator) entrega una evaluación en la escala de 1.0 a 10 al sitio evaluado.

\section{Recomendaciones y Principios de la WAI5}

Estas recomendaciones se agrupan de acuerdo a 4 Principios que definen a un sitio Web accesible, estos son:

Perceptible: significa que el usuario debe poder acceder a la información y que esté disponible para los sentidos (visión y audición principalmente).

Operable: que el usuario debe poder navegar por la web.

Comprensible: que debe entenderse la información que hay contenida en la página web.

Robusto: que la accesibilidad no debe depender de la tecnología empleada para acceder a la página web.

Cada recomendación contiene uno o más puntos de verificación que explican cómo ésta debe ser aplicada, y a su vez, cada punto tiene asignada una prioridad que indica el impacto del punto de verificación en la accesibilidad del sitio.

Considerando la normativa vigente en Chile, de acuerdo al Decreto №1, emanado por el Ministerio Secretaría General de la Presidencia en junio de 2015, que aprueba la norma técnica sobre sistemas y sitios web de los órganos de la administración del Estado, en relación al desarrollo e implementación de sitios web accesibles, cumpliendo las normas vigentes existentes, en específico lo recomendado por el W3C (W3C, 2018b) como estándar internacional, se indica que el nivel idóneo de conformidad de accesibilidad corresponde a doble $A(A A)$, lo que implica que un sitio web debe cumplir con 37 criterios de conformidad. Para cada pauta se proporcionan criterios de conformidad verificables, definiéndose tres niveles de conformidad: A (el más bajo), AA y AAA (el más alto).

Del conjunto de prioridades en el sitio, se definen tres niveles de conformidad:

- $\quad$ Nivel A: Todos los puntos de control de prioridad 1 son cumplidos.

- Nivel AA: Todos los puntos de control de prioridades 1 y 2 son cumplidos.

\footnotetext{
${ }^{5}$ the Web Accessibility Initiative of the W3C
} 
- Nivel AAA: Todos los puntos de control de prioridades 1, 2 y 3 son cumplidos.

Considerando los criterios de conformidad de la WAI (Universidad de Alicante, 2018) se establecen una cantidad de criterios de acuerdo al Nivel de conformidad, quedando para los niveles $A$ y AA de la siguiente forma:

- Criterios de conformidad Nivel A: 25 criterios

- Criterios de conformidad Nivel AA: 13 criterios

Esto da un total de 38 criterios de conformidad que deben cumplir los sitios chilenos para cumplir con la normativa establecida, uno más de lo indicado en la "Guía técnica para la implementación de sitios Web Accesible" (pág. 10; pág. 15) elaborada por la Coordinación de Accesibilidad - Servicio Nacional de la Discapacidad Chileno, año 2017.

\section{Universidades del Consejo de Rectores}

El Consejo de Rectores de las Universidades Chilenas (CRUCH) se crea el 14 de agosto del año 1954, en virtud a lo establecido en la Ley $\mathrm{N}^{\circ} 11.575$, artículo 36, letra c, que define al $\mathrm{CRUCH}$ como un organismo de coordinación de la labor universitaria de la nación. Se le asigna también la tarea de mejorar el rendimiento y calidad de la enseñanza universitaria a través de un trabajo propositivo ${ }^{6}$.

En la actualidad, el Consejo de Rectores de las Universidades Chilenas está integrado por los rectores de las veintisiete universidades estatales y no estatales con vocación pública del país y lo preside el Ministro(a) de Educación. Estas universidades poseen amplia trayectoria y excelencia en Educación Superior, con presencia en todas las regiones de Chile.

Considerando la relevancia de este consejo para el desarrollo académico y/o científico del país se abordan sus sitios web, en específico las páginas iniciales como puerta de entrada virtuales a las universidades, evidenciando su peso desde el punto de vista informativo y de consulta por parte de los agentes involucrados en el proceso educativo, profesores, estudiantes, profesionales de diversas áreas, en la Tabla 1 se presenta el listado de estas universidades con sus direcciones web.

Tabla 1. Sitios web seleccionados, Universidades del Consejo de Rectores, para el análisis de la accesibilidad web.

\begin{tabular}{|c|c|c|}
\hline & Universidad & Sitio web \\
\hline 1. & Universidad de Chile & http://www.uchile.cl/ \\
\hline 2. & Pontificia Universidad Católica de Chile & http://www.uc.cl/ \\
\hline 3. & Universidad de Concepción & http://www.udec.cl/ \\
\hline 4. & Pontificia Universidad Católica de Valparaíso & http://www.ucv.cl/ \\
\hline 5. & Universidad Técnica Federico Santa María & http://www.utfsm.cl/ \\
\hline 6. & Universidad de Santiago de Chile & http://www.usach.cl/ \\
\hline 7. & Universidad Austral de Chile & http://www.uach.cl/ \\
\hline 8. & Universidad Católica del Norte & http://www.ucn.cl/ \\
\hline 9. & Universidad de Valparaíso & http://www.uv.cl/ \\
\hline 10. & Universidad de Antofagasta & http://www.uantof.cl/ \\
\hline 11. & Universidad de La Serena & http://www.userena.cl/ \\
\hline 12. & Universidad del Bio Bio & http://www.ubiobio.cl/ \\
\hline 13. & Universidad de La Frontera & http://www.ufro.cl/ \\
\hline 14. & Universidad de Magallanes & http://www.umag.cl/ \\
\hline 15. & Universidad de Talca & http://www.utalca.cl/ \\
\hline 16. & Universidad de Atacama & http://www.uda.cl/ \\
\hline
\end{tabular}

\footnotetext{
${ }^{6}$ http://www.consejoderectores.cl/historia
} 
17. Universidad de Tarapacá

18. Universidad Arturo Prat

19. Universidad Metropolitana de Ciencias de la Educación

20. Universidad de Playa Ancha

21. Universidad Tecnológica Metropolitana

22. Universidad de Los Lagos

23. Universidad Católica del Maule

24. Universidad Católica de la Santísima Concepción

25. Universidad Católica de Temuco

26. Universidad de Aysén

27. Universidad de O'Higgins http://www.uta.cl/

http://www.unap.cl/

http://www.umce.cl/

http://www.upla.cl/

http://www.utem.cl/

http://www.ulagos.cl

http://portal.ucm.cl/

http://www.ucsc.cl/

https://uct.cl/

http://www.uaysen.cl/

https://www.uoh.cl/

\section{Resultados}

Metodología de trabajo usando herramientas de análisis automático TAW y eXaminator:

Herramienta TAW. Utilizando las herramientas específicas que permiten esbozar el grado de accesibilidad de una página web, recomendadas para tal efecto por la WAI, como es la herramienta TAW o Test de Accesibilidad de la Web (CTIC, 2018), detectando los principales errores de la página principal en el nivel $A A$ atendiendo la normativa exigida en Chile. Los resultados se presentan en la tabla 2.

La metodología de aplicación de la herramienta TAW es el ingreso del URL indicando el nivel específico de análisis y las tecnologías soportadas, luego la herramienta genera un documento resumen que muestra el total de los problemas (son necesarias correcciones), las advertencias (deben revisarse manualmente) y los puntos no verificados (que requieren un análisis manual completo) y los organiza por cada principio (Perceptible, Operable, Comprensible y Robusto).

Tabla 2. Resultados de la evaluación de los sitios de las universidades del Consejo de Rectores con TAW nivel AA. con los problemas, advertencias y no verificables de cada página inicial por universidad del

\begin{tabular}{|c|c|c|c|c|}
\hline $\mathrm{N}^{\circ}$ & Universidad & Problemas & Advertencias & No verificados \\
\hline 1. & Universidad de Chile & $5,5 \%$ & $89,6 \%$ & $4,9 \%$ \\
\hline 2. & Pontificia Universidad Católica de Chile & $10,2 \%$ & $85,9 \%$ & $3,9 \%$ \\
\hline 3. & Universidad de Concepción & $3,5 \%$ & $95,4 \%$ & $1,1 \%$ \\
\hline 4. & $\begin{array}{l}\text { Pontificia Universidad Católica de } \\
\text { Valparaíso }\end{array}$ & $9,3 \%$ & $85,8 \%$ & $4,8 \%$ \\
\hline 5. & $\begin{array}{l}\text { Universidad Técnica Federico Santa } \\
\text { María }\end{array}$ & $17,0 \%$ & $67,9 \%$ & $15,2 \%$ \\
\hline 6. & Universidad de Santiago de Chile & $9,0 \%$ & $78,2 \%$ & $12,8 \%$ \\
\hline 7. & Universidad Austral de Chile & $11,7 \%$ & $83,7 \%$ & $4,6 \%$ \\
\hline 8. & Universidad Católica del Norte & $21,2 \%$ & $75,6 \%$ & $3,2 \%$ \\
\hline 9. & Universidad de Valparaíso & $37,0 \%$ & $56,2 \%$ & $6,8 \%$ \\
\hline 10. & Universidad de Antofagasta & $12,8 \%$ & $84,0 \%$ & $3,3 \%$ \\
\hline 11. & Universidad de La Serena & $3,6 \%$ & $95,1 \%$ & $1,4 \%$ \\
\hline 12. & Universidad del Bio Bio & $35,5 \%$ & $60,0 \%$ & $4,5 \%$ \\
\hline 13. & Universidad de La Frontera & $9,9 \%$ & $86,0 \%$ & $4,0 \%$ \\
\hline 14. & Universidad de Magallanes & $8,1 \%$ & $81,5 \%$ & $10,5 \%$ \\
\hline 15. & Universidad de Talca & $37,1 \%$ & $57,7 \%$ & $5,2 \%$ \\
\hline 16. & Universidad de Atacama & $4,0 \%$ & $94,8 \%$ & $1,2 \%$ \\
\hline 17. & Universidad de Tarapacá & $7,7 \%$ & $86,7 \%$ & $5,5 \%$ \\
\hline 18. & Universidad Arturo Prat & $18,8 \%$ & $71,6 \%$ & $9,7 \%$ \\
\hline 19. & $\begin{array}{l}\text { Universidad Metropolitana de Ciencias } \\
\text { de la Educación }\end{array}$ & $6,1 \%$ & $92,0 \%$ & $1,9 \%$ \\
\hline 20. & Universidad de Playa Ancha & $16,2 \%$ & $81,7 \%$ & $2,0 \%$ \\
\hline 21. & Universidad Tecnológica Metropolitana & $13,0 \%$ & $82,2 \%$ & $4,8 \%$ \\
\hline 22. & Universidad de Los Lagos & $39,6 \%$ & $52,7 \%$ & $7,7 \%$ \\
\hline 23. & Universidad Católica del Maule & $29,2 \%$ & $65,7 \%$ & $5,1 \%$ \\
\hline 24. & $\begin{array}{l}\text { Universidad Católica de la Santísima } \\
\text { Concepción }\end{array}$ & $21,5 \%$ & $74,5 \%$ & $4,0 \%$ \\
\hline
\end{tabular}




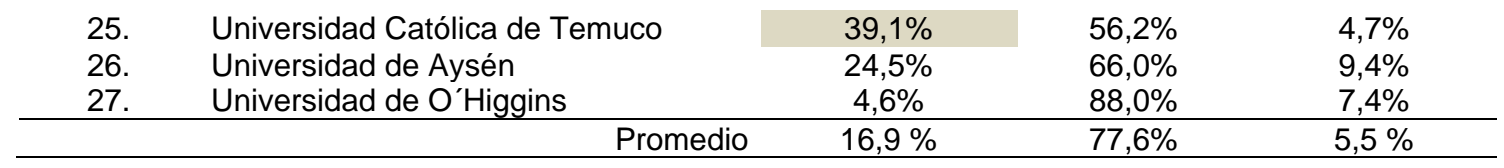

\section{Análisis Evaluación automática TAW}

La evaluación usando TAW arroja que todas las universidades presentan problemas, advertencias y no verificados, es decir son sitios no accesibles. Si se considera que los "problemas" son señalados por TAW como imperativo de correcciones, el porcentaje promedio es importante, $16,9 \%$, destacando cinco universidades con errores sobre el $30 \%$.

Tabla 3. Presenta el porcentaje de Problemas encontrados en base a los cuatro principios: Perceptible, Operable, Comprensible, Robusto, con ellos se obtiene el porcentaje de cada uno.

\begin{tabular}{|c|c|c|c|c|c|}
\hline $\mathrm{N}^{\circ}$ & Universidad & Perceptible & Operable & Comprensible & Robusto \\
\hline 1. & Universidad de Chile & $50,8 \%$ & $35,0 \%$ & $1,9 \%$ & $12,3 \%$ \\
\hline 2. & $\begin{array}{l}\text { Pontificia Universidad Católica de } \\
\text { Chile }\end{array}$ & $66,2 \%$ & $21,6 \%$ & $3,3 \%$ & $8,9 \%$ \\
\hline 3. & Universidad de Concepción & $8,4 \%$ & $4,0 \%$ & $1,7 \%$ & $86,0 \%$ \\
\hline 4. & $\begin{array}{l}\text { Pontificia Universidad Católica de } \\
\text { Valparaíso }\end{array}$ & $51,2 \%$ & $38,6 \%$ & $3,6 \%$ & $6,6 \%$ \\
\hline 5. & $\begin{array}{l}\text { Universidad Técnica Federico } \\
\text { Santa María }\end{array}$ & $47,3 \%$ & $27,7 \%$ & $17,0 \%$ & $8,0 \%$ \\
\hline 6. & Universidad de Santiago de Chile & $45,9 \%$ & $43,6 \%$ & $8,3 \%$ & $2,3 \%$ \\
\hline 7. & Universidad Austral de Chile & $73,0 \%$ & $20,6 \%$ & $4,6 \%$ & $1,8 \%$ \\
\hline 8. & Universidad Católica del Norte & $17,4 \%$ & $11,9 \%$ & $7,9 \%$ & $62,8 \%$ \\
\hline 9. & Universidad de Valparaíso & $57,1 \%$ & $34,7 \%$ & $5,5 \%$ & $2,7 \%$ \\
\hline 10. & Universidad de Antofagasta & $27,3 \%$ & $22,3 \%$ & $3,0 \%$ & $47,4 \%$ \\
\hline 11. & Universidad de La Serena & $14,4 \%$ & $4,4 \%$ & $0,6 \%$ & $80,5 \%$ \\
\hline 12. & Universidad del Bio Bio & $42,3 \%$ & $46,0 \%$ & $4,5 \%$ & $7,2 \%$ \\
\hline 13. & Universidad de La Frontera & $66,7 \%$ & $15,1 \%$ & $5,4 \%$ & $12,9 \%$ \\
\hline 14. & Universidad de Magallanes & $41,1 \%$ & $23,4 \%$ & $8,9 \%$ & $26,6 \%$ \\
\hline 15. & Universidad de Talca & $51,0 \%$ & $21,0 \%$ & $11,5 \%$ & $16,4 \%$ \\
\hline 16. & Universidad de Atacama & $23,5 \%$ & $6,1 \%$ & $0,9 \%$ & $69,4 \%$ \\
\hline 17. & Universidad de Tarapacá & $67,2 \%$ & $18,5 \%$ & $4,4 \%$ & $10,0 \%$ \\
\hline 18. & Universidad Arturo Prat & $32,4 \%$ & $26,7 \%$ & $3,4 \%$ & $37,5 \%$ \\
\hline 19. & $\begin{array}{l}\text { Universidad Metropolitana de } \\
\text { Ciencias de la Educación }\end{array}$ & $26,3 \%$ & $5,5 \%$ & $2,6 \%$ & $65,5 \%$ \\
\hline 20. & Universidad de Playa Ancha & $27,6 \%$ & $30,5 \%$ & $3,0 \%$ & $38,9 \%$ \\
\hline 21. & $\begin{array}{l}\text { Universidad Tecnológica } \\
\text { Metropolitana }\end{array}$ & $34,3 \%$ & $49,7 \%$ & $8,1 \%$ & $7,8 \%$ \\
\hline 22. & Universidad de Los Lagos & $27,5 \%$ & $24,2 \%$ & $9,2 \%$ & $39,1 \%$ \\
\hline 23. & Universidad Católica del Maule & $31,2 \%$ & $34,6 \%$ & $3,4 \%$ & $30,9 \%$ \\
\hline 24. & $\begin{array}{l}\text { Universidad Católica de la } \\
\text { Santísima Concepción }\end{array}$ & $37,0 \%$ & $43,5 \%$ & $3,0 \%$ & $16,5 \%$ \\
\hline 25. & Universidad Católica de Temuco & $39,9 \%$ & $27,6 \%$ & $5,5 \%$ & $27,0 \%$ \\
\hline 26. & Universidad de Aysén & $23,3 \%$ & $59,7 \%$ & $15,1 \%$ & $1,9 \%$ \\
\hline 27. & Universidad de O'Higgins & $26,9 \%$ & $20,8 \%$ & $2,8 \%$ & $49,5 \%$ \\
\hline & Promedio & $39,2 \%$ & $26,6 \%$ & $5,5 \%$ & $28,8 \%$ \\
\hline
\end{tabular}

\section{Análisis según principio}

Los principios permiten orientar la evaluación en aquellos aspectos relevantes para una accesibilidad integral, el 39,2 \% de problemas del principio Perceptible es relevante considerando que significa que el usuario debe poder acceder a la información y que esté disponible para los sentidos (visión y audición principalmente). Luego le sigue es escala de problemas el principio Robusto, con un 28,8 \% de errores. Este principio se 
relaciona con que la accesibilidad no debe depender de la tecnología empleada para acceder a la página web. Sin duda ambos principios cobran especial relevancia si pensamos en un usuario con alguna discapacidad.

\section{Evaluación con eXaminator}

\section{¿Qué es eXaminator?}

Es un servicio en línea para evaluar de modo automático la accesibilidad de una página web, usando como referencia algunas técnicas recomendadas por las Pautas de Accesibilidad para el Contenido Web 2.0 (WCAG 2.0). Adjudica una puntuación entre 1 y 10 como un indicador rápido de la accesibilidad de las páginas y proporciona un informe detallado de las pruebas realizadas. (Benavidez, 2005)

Tabla 4. Resultados análisis herramienta eXaminator

\begin{tabular}{|c|c|c|c|c|c|c|}
\hline \multirow[t]{2}{*}{ Universidad } & \multirow[t]{2}{*}{ Evaluación } & \multicolumn{5}{|c|}{ Resultados generales de pruebas } \\
\hline & & Excelente & Muy bien & Regular & Mal & Muy Mal \\
\hline Universidad de Chile & 5,6 & 7 & 1 & 4 & 4 & 3 \\
\hline $\begin{array}{l}\text { Pontificia Universidad } \\
\text { Católica de Chile }\end{array}$ & 4,2 & 7 & 1 & 3 & 10 & 6 \\
\hline Universidad de Concepción & 4,3 & 6 & 0 & 4 & 4 & 10 \\
\hline $\begin{array}{l}\text { Pontificia Universidad } \\
\text { Católica de Valparaíso }\end{array}$ & 4,7 & 6 & 0 & 5 & 6 & 3 \\
\hline $\begin{array}{l}\text { Universidad Técnica } \\
\text { Federico Santa María }\end{array}$ & \multicolumn{6}{|c|}{ Unknown SSL protocol error in connection to www.utfsm.cl:443 } \\
\hline $\begin{array}{l}\text { Universidad de Santiago de } \\
\text { Chile }\end{array}$ & \multicolumn{6}{|c|}{ Unknown SSL protocol error in connection to www.usach.cl:443 } \\
\hline Universidad Austral de Chile & 4,1 & 5 & 0 & 2 & 5 & 8 \\
\hline $\begin{array}{l}\text { Universidad Católica del } \\
\text { Norte }\end{array}$ & 5,1 & 9 & 0 & 2 & 4 & 7 \\
\hline Universidad de Valparaíso & 4,4 & 5 & 1 & 1 & 4 & 5 \\
\hline Universidad de Antofagasta & 4,3 & 4 & 0 & 2 & 5 & 4 \\
\hline Universidad de La Serena & 4,6 & 6 & 1 & 5 & 9 & 4 \\
\hline Universidad del Bio Bio & 4,5 & 7 & 0 & 2 & 8 & 7 \\
\hline Universidad de La Frontera & \multicolumn{6}{|c|}{ Unknown SSL protocol error in connection to www.ufro.cl:443 } \\
\hline Universidad de Magallanes & 5,3 & 7 & 1 & 4 & 4 & 4 \\
\hline Universidad de Talca & 5,7 & 3 & 0 & 0 & 4 & 0 \\
\hline Universidad de Atacama & 5,9 & 9 & 1 & 4 & 3 & 5 \\
\hline Universidad de Tarapacá & \multicolumn{6}{|c|}{ Unknown SSL protocol error in connection to www.uta.cl:443 } \\
\hline Universidad Arturo Prat & 5,2 & 3 & 0 & 0 & 5 & 0 \\
\hline $\begin{array}{l}\text { Universidad Metropolitana } \\
\text { de Ciencias de la Educación }\end{array}$ & 4,1 & 6 & 1 & 3 & 9 & 6 \\
\hline Universidad de Playa Ancha & 5,5 & 3 & 0 & 0 & 4 & 0 \\
\hline $\begin{array}{l}\text { Universidad Tecnológica } \\
\text { Metropolitana }\end{array}$ & 5,8 & 8 & 0 & 2 & 7 & 2 \\
\hline Universidad de Los Lagos & 4,4 & 7 & 1 & 2 & 5 & 7 \\
\hline $\begin{array}{l}\text { Universidad Católica del } \\
\text { Maule }\end{array}$ & 6.3 & 8 & 0 & 2 & 2 & 5 \\
\hline $\begin{array}{l}\text { Universidad Católica de la } \\
\text { Santísima Concepción }\end{array}$ & 6.5 & 10 & 1 & 2 & 2 & 6 \\
\hline $\begin{array}{l}\text { Universidad Católica de } \\
\text { Temuco }\end{array}$ & \multicolumn{6}{|c|}{ Unknown SSL protocol error in connection to uct.cl:443 } \\
\hline Universidad de Aysén & \multirow{2}{*}{\multicolumn{6}{|c|}{$\begin{array}{c}\text { Unknown SSL protocol error in connection to www.uaysen.cl:443 } \\
\text { Unknown SSL protocol error in connection to www.uoh.cl:443 }\end{array}$}} \\
\hline Universidad de O'Higgins & & & & & & \\
\hline
\end{tabular}

\section{Análisis eXaminator}

Al analizar los resultados logrados por la evaluación automática con eXaminator se observa el bajo nivel de evaluación general logrado por el gran porcentaje de sitios, las que logran la mayor evaluación, muy discreta por lo de demás, son la Universidad 
Católica del Maule $(6,3)$ y la Universidad Católica de la Santísima Concepción $(6,5)$, considerando que esta evaluación va de 1.0 a 10.

Los fallos más frecuentes tienen relación con ausencia de alternativa textual tanto en enlaces como en imágenes. Otra deficiencia recurrente en la presencia de enlaces con el mismo texto pero con diferentes destinos.

Estas deficiencias afectan directamente a personas no videntes que usan herramientas de apoyo para recorrer los sitios web siendo una barrera estas carencias considerando que un enlace puede llevar a otro sitio desconocido y generar pérdida de tiempo y desorientación, mientras que las imágenes sin texto alternativo no son comprendidas por los lectores por tanto su aporte es nulo.

Es importante destacar que siete universidades no pudieron ser evaluadas porque la herramienta de evaluación eXaminator no identifico la dirección ingresada, desconociéndose la razón.

\section{Discusión}

Existe consenso en cuanto a considerar internet un espacio de información y comunicación por excelencia en la sociedad actual, razón por la cual las instituciones, independientes de sus objetivos o características, deben ser capaces de hacer accesible su información a todo usuario que así lo requiera, independiente de sus capacidades físicas o intelectuales. En Chile hay evidencias previas como el denominado "Desarrollo de Sitios Web: la ley, el orden y los estándares" (Figueroa Valdés, 2007) que evidencian la importancia de esta porque se fortalecen la inclusión y disminuyen las barreras hacia el acceso a la información, para llegar a una Internet democrática con acceso igualitario a la información sin importar los medios por los cuales se accede a ella. Del mismo estudio se destaca que mencionan que los sitios web chilenos carecen de un nivel de estandarización.

Chile tiene políticas que establecen la igualdad de oportunidades e inclusión social en personas con discapacidad, Ley 20.422, junto a leyes más precisas como es el Decreto $N^{\circ} 1$ de junio de 2015 que aprueba norma técnica sobre sistemas y sitios web de los órganos de la administración del estado y la Guía técnica para la implementación de sitios Web Accesibles establecida por el Servicio Nacional de la Discapacidad para orientar el desarrollo de sitios web. Esto deja en evidencia a Chile en su liderazgo latinoamericano en cuando a inclusión de Internet con normas internacionales como los estándares entregados por el World Wide Web Consortium en sus Pautas de Accesibilidad para el Contenido Web (WCAG) 2.0., señalando que el nivel idóneo de conformidad de accesibilidad corresponde a doble A (AA).

En paralelo hay investigaciones que buscan sistematizar las diferentes legislaciones vigentes en torno al tema de accesibilidad web, es así como se menciona el estudio denominado Legislación sobre accesibilidad web: una comparativa de seis países, el cual abarca España, Uruguay, Colombia, Ecuador, Brasil y Chile, realizado por Peñafiel y Luján en el año 2014, el cual da cuenta de ser un estudio preliminar del estado de la legislación sobre accesibilidad web de algunos países. Su objetivo está centrado en establecer el estado de la legislación web entre los países seleccionados tomando como parámetros: el reconocimiento de la legislación a nivel internacional y local así como la identificación de los progresos obtenidos por cada país investigado en función de los 
estándares de la W3C y la WAI7, las Pautas de Accesibilidad al Contenido Web (WCAG) 1.0 y 2.0, y los órganos reguladores. (Peñafiel \& Luján Mora, 2014)

Existen evidencias de esfuerzos por sistematizar lo relacionado con accesibilidad a nivel de Iberoamérica, en un estudio de la Universidad de Alicante que refrenda lo relativo a accesibilidad web, abordando la legislación en torno al tema de 11 países iberoamericanos, incluyendo a Chile. (Luján, 2018)

Comprendiendo la relevancia de actualizar los antecedentes sobre legislación y evaluación de accesibilidad web a nivel universitario se analizaron los sitios iniciales de las universidades del Consejo de Rectores, considerando su relevancia y presencia a nivel nacional, para esto se usaron dos herramientas específicas que permiten esbozar el grado de accesibilidad de una página web, recomendadas para tal efecto por la WAI, como es la herramienta TAW y eXaminator, lo que permitió enfrentar los resultados, de esta forma se logró precisar cuáles son las debilidades más recurrentes en su diseño y que afectan directamente el nivel de accesibilidad.

Todos los sitios analizados no son accesibles, siendo las debilidades más recurrentes el no contar con alternativas textuales para elementos como imágenes, videos, animaciones $u$ otros que lo exigen, junto a esto los enlaces no especifican claramente su destino y presentan enlaces idénticos con diferentes destinos lo que desorienta la navegación de los usuarios que usan alguna herramienta de apoyo para la lectura de estos espacios.

La investigación denominada Accesibilidad en Portales Universitarios Chilenos realizada por Claudia Vargas, Arturo Sepúlveda, Roberto Muñoz, Eliana Providel de la Escuela de Ingeniería Civil Informática, Facultad de Ingeniería de la Universidad de Valparaíso desarrollada el año 2012, sirve de referencia inicial para vislumbrar que es un tema de interés hace tiempo y que no existen estudios posteriores que aborden la legislación vigente o que busquen actualizar el proceso de evaluación de las web universitarias. (Vargas \& Sepúlveda, 2012) los autores señalan que su trabajo es el primer acercamiento para determinar el grado de accesibilidad de los portales universitarios a nivel nacional. Los resultados muestran que después de la evaluación a las cinco principales páginas de 58 portales universitarios que existen en Chile, el 100 por ciento de los evaluados no cumplen con el grado mínimo de accesibilidad propuesto por la Wordl Wide Web Consortium (W3C).

Esta investigación es preliminar considerando que se utilizaron herramientas automáticas de evaluación, las cuales deberían ser corroboradas en forma manual, además de realizar una evaluación con alguna o algunas personas que presentan algún tipo de discapacidad, de igual forma establece bases para estudios posteriores que permitan una evaluación con mayor profundidad de instituciones educativas no solo a nivel universitario, considerando el rol trascendental que cumplen para el desarrollo y avance de un país, porque la accesibilidad junto con ser un derecho no es un tema exclusivo de personas con alguna discapacidad sino que el beneficio es para toda la población.

\footnotetext{
${ }^{7}$ https://www.w3.org/WAl/
} 


\section{Referencias}

Benavidez, C. (2005). eXaminator. Retrieved from http://examinator.ws/

COCEMFE. (2018). Observatorio de la Accesibilidad. Retrieved from https://www.observatoriodelaaccesibilidad.es/elobservatorio/

CTIC. (2018). TAW | Servicios de accesibilidad y movilidad web. Centro Tecnológico.

Discapnet. (2018). La accesibilidad Web | Discapnet. Retrieved from https://www.discapnet.es/areas-tematicas/accesibilidad

Fernández, S., \& Pértegas, S. (2002). Investigación cuantitativa y cualitativa. Cadena Atención Primaria, 9(Figura 1), 76-78.

Figueroa Valdés, F. (2007). Desarrollo de Sitios Web: la ley, el orden y los estándares. Serie Bibliotecología Y Gestión de Información, (28), 1-24.

I. Barberis. (2018). EyN: Chile es calificado como el mejor país latinoamericano en acceso y uso de internet. Retrieved from http://www.economiaynegocios.cl/noticias/noticias. asp?id=44 8731

Luján, S. (2018). Universidad de Alicante. Accesibilidad Web: Legislación. Retrieved from

http://accesibilidadweb.dlsi.ua.es/?menu=legislacion

Luján Mora, S. (2018). Herramientas de revisión automática de la accesibilidad web. Retrieved from

http://accesibilidadweb.dlsi.ua.es/?menu=hr-revisionautomaticas

ONCE. (2018). Accesibilidad | Fundación ONCE para la Cooperación e Inclusión Social de Personas con Discapacidad. Retrieved from https://www.fundaciononce.es/
Pastor, C. (2012). Aportaciones del Diseño Universal para el Aprendizaje y de los materiales digitales en el logro de una enseñanza accesible. Diversidad.Murciaeduca.Es, 1-13.

Peñafiel, M., \& Luján Mora, S. (2014). Legislación sobre accesibilidad web: una comparativa de seis países. Revista Politécnica, 34(2), 34-45.

RAE. (2018). DLE: accesible - Diccionario de la lengua española Edición del Tricentenario. Retrieved from https://dle.rae.es/?id=0K2DI9N

SENADIS. (2018). Servicio Nacional de la Discapacidad. Retrieved from https://www.senadis.gob.cl/

Sidar. (2018). Principios del Diseño Universal o Diseño para Todos. Retrieved from http://www.sidar.org/recur/desdi/usable/dudt.php

Universidad de Alicante. (2018). Criterios de conformidad accesibilidad WGAC. Retrieved from http://accesibilidadweb.dlsi.ua.es/?menu=criterios -2.0

Vargas, C., \& Sepúlveda, A. (2012). Accesibilidad en Portales Universitarios Chilenos. XXIV Encuentro Chileno .... (December).

W3C. (2018a). Web Accessibility Initiative (WAI) - home page | Web Accessibility Initiative (WAI) | W3C. Retrieved from https://www.w3.org/

W3C. (2018b). World Wide Web Consortium (W3C). Retrieved from https://www.w3.org/ 